\title{
Q Fever Outbreaks in Dalmatia, Croatia
}

\author{
Miro Morovic ${ }^{1, *}$, Nedjeljka Babic Milutin ${ }^{2}$, Duska Grgić ${ }^{3}$ and Oktavija Djakovic Rode ${ }^{4}$
}

${ }^{I}$ Zadar General Hospital, Department for Infectious Diseases, Zadar; ${ }^{2}$ Sibenik General Hospital, Department for Infectious Diseases, Sibenik; ${ }^{3}$ Split University Hospital, Department for Infectious Diseases, Split and ${ }^{4}$ University Hospital for Infectious Diseases, Serologic laboratory, Zagreb

\begin{abstract}
The aim of the study was to describe the clinical and epidemiological features of the first greater outbreaks of Q fever in Dalmatia, Croatia. In 2003, January to April, 97 patients with Q fever pneumonia were hospitalized in three Dalmatian hospitals - Zadar, Sibenik and Split. In 2004, January to May, the outbreak involved 100 hospitalized patients. Many cases were epidemiologically connected and came from the well-known endemic foci but a number of sporadic cases among strictly urban population was also noted. During the outbreaks period antibodies to Coxiella burnetii were detected in $10.8 \%$ of 1378 domestic animals from the main foci. Since during this period nothing unusual happened in the sheep and goat flocks and in the windborne spread of coxiella spores, other risk factors may have contributed to these changes.
\end{abstract}

Key Words: Q fever, outbreaks, Croatia.

\section{INTRODUCTION}

Q fever has been a well-known and mandatory noticeable disease in Croatia since 1954; it occurs usually sporadically in areas predominantly rich with sheep and goats [1-4]. The notification was based on serologic diagnosis performed until the nineties on the complement fixation $\mathrm{CF}$ test and after that on indirect immunofluorescence assay (IFA) and/or enzyme immunoassay (EIA) tests.

The mean annual incidence of $\mathrm{Q}$ fever in Croatia from 1994 to 2002 was 0.87 reported cases per 100,000 population, but in 2003 and 2004 this incidence rose to 3.44 per 100,000 . This change in epidemiology was predominantly due to two large outbreaks in North and Mid Dalmatia, the central part of the Adriatic coast of Croatia, with 197 people treated in hospitals for serologically confirmed $\mathrm{Q}$ fever pneumonia.

We report an investigation into clinical and epidemiological characteristics of these Q fever outbreaks, as well as the factors which influenced them.

\section{PATIENTS AND METHODS}

A retrospective study was carried out by experienced specialists on all the patients with $\mathrm{Q}$ fever pneumonia hospitalized in the three Dalmatian hospitals-Zadar, Sibenik, Split -during a 6-year period (January 2001-December 2006).

The County Hospitals Ethic Committees' approvals for the study, and the written informed consents from the patients were duly obtained.

*Address correspondence to this author at the Zadar General Hospital, Department for Infectious Diseases, Zadar;

E-mail: miro.morovic@zd.t-com.hr

\section{Demographic, Geographic and Meteorologic Data}

The study area, North and Mid Dalmatia, which covers the central part of the Eastern Adriatic, consists of three counties: the Zadar County in North Dalmatia, and the Sibenik and Split Counties in Mid Dalmatia (Fig. 1).

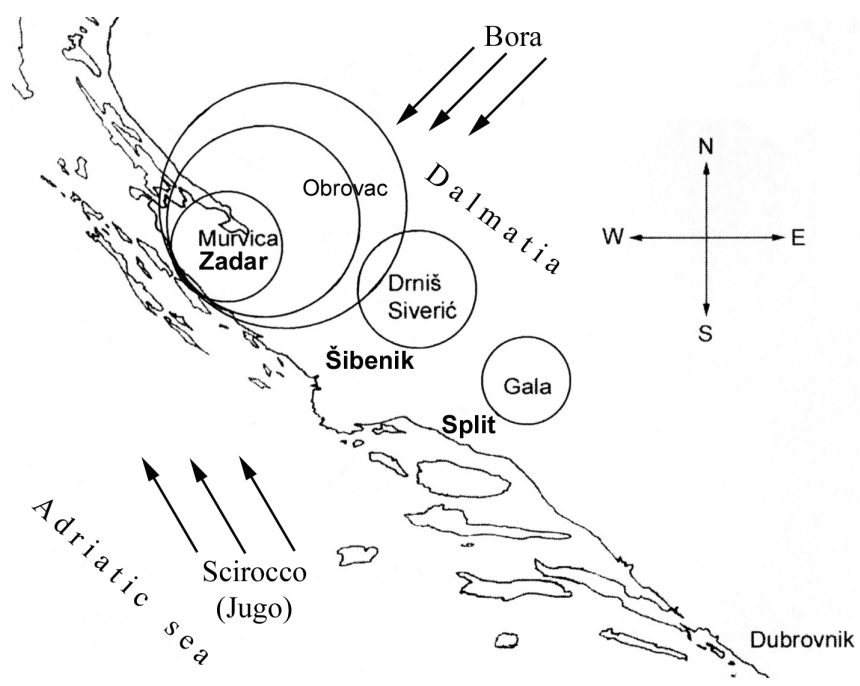

Fig. (1). Location of cases of Q fever (circles) in Dalmatia, Croatia, 2003/4. Direction of the main winds during the outbreaks period are shown; the maximum speed of Bora was $9.3 \mathrm{~m} / \mathrm{s}$ (moderate speed) and of Scirocco (Jugo) $12.3 \mathrm{~m} / \mathrm{s}$ (high speed).

This area of $15,570 \mathrm{~km}^{2}$ had 711,612 inhabitants in 2001 . The climate is typical Mediterranean: with small temperature variations, dry winters and springs, rainfall mainly during autumn and an average relative humidity about $66 \%$. 
Meteorologic data were obtained from the Croatian Meteorological Institute, Zagreb. Time series (at 07 h, 14 h and $21 \mathrm{~h}$ local time) of instantaneous wind data and daily precipitation values have been used.

Sheep and goats breeding is traditional throughout the North and Mid Dalmatia. The main lambing and goating season is from late December to May with the peak time in February.

\section{The 2003 Outbreak}

The number of patients with $Q$ fever pneumonia hospitalized from January to April was as follows:

- The thirty four patients in the Split University Hospital, mostly from January 30 to February 15, 2003, included: fourteen patients from Gala, a small rural village known for a long time as a $\mathrm{Q}$ fever endemic area in the northern part of the Split County and nineteen patients from the group of roadworkers working in the Obrovac area, also a well-known $\mathrm{Q}$ fever focus in the northern part of the Zadar County (Fig. 1).

- The twenty four patients in the Sibenik General Hospital, most of them from February 17 to March 31, 2003, included fourteen patients from the Siveric-Drnis rural area, known as an old Q fever area in the northern part of the Sibenik County and four patients from the mentioned group of road-.workers already mentioned.

- The thirty nine patients in the Zadar General Hospital, most of them between February 17 and 25, 2003, included seventeen patients from the road-workers group.

Other hospitalized patients had no epidemiological connection with these outbreaks and were considered sporadical.

No Q fever relevant epidemiological data were registered in the 9 patients who lived in strictly urban area (6 in Zadar, 4 in Sibenik and 1 in Split).

Acute Q fever was serologically confirmed in all 97 hospitalized patients.

\section{The 2004 Outbreak}

The details of number of patients with Q fever pneumonia hospitalized from January to May were as follows:

- The seventy eight patients in the Zadar General Hospital, mostly from March 3 to March 24, 2004, included eleven workers in an aluminium factory in the Murvica area, placed very close to the city of Zadar, an old Q fever focus with numerous pastures and sheep farms (Fig. 1).

- The seventeen patients in the Sibenik General Hospital, most of them from March 14 to March 31, 2004, of which 8 from the Siveric-Drnis area.

- Only five patients were hospitalized in the Split University Hospital, of which 3 were from Gala.

All the cases were considered sporadic except those from the factory mentioned above.

No Q fever relevant epidemiological data were registered in twenty one patients who lived in strictly urban area (18 patients in Zadar, 2 in Sibenik and 1 in Split).
Acute Q fever was serologically confirmed in all 100 hospitalized patients.

The number of patients and the places they come from are summarized in Table $\mathbf{1}$

Table 1.

\begin{tabular}{|c|c|c|}
\hline \multirow{2}{*}{ County/place } & \multicolumn{2}{|c|}{ Year } \\
\cline { 2 - 3 } & $\mathbf{2 0 0 3}$ & $\mathbf{2 0 0 4}$ \\
\hline \hline Split & 14 & 3 \\
\hline Gala & 19 & - \\
\hline Obrovac* & 1 & 2 \\
\hline Others & 14 & 8 \\
\hline Sibenik & 4 & 9 \\
\hline Siveric-Drnis & 6 & 11 \\
\hline Obrovac* & & 67 \\
\hline Others & 17 & \\
\hline Zadar & & \\
\hline Obrovac* & 22 & \\
\hline Murvica & & \\
\hline Others & & \\
\hline
\end{tabular}

* Group of 40 road-workers from Obrovac, region in the northern part of the Zada County.

\section{Serologic Diagnosis}

Acute and convalescent serum samples from patients were transported to the University Hospital for Infectious Diseases in Zagreb where the serologic diagnosis was performed by enzyme-linked immunosorbent assay (ELISA) (Virion-Serion, Germany). Antibodies IgM to C.burnetii phase 2 , and $\operatorname{IgG}$ and $\operatorname{IgA}$, phase 1 were determined by comparing the absorbance value of unknown samples with that of the cut-off control value. The diagnosis of $Q$ fever was confirmed by seroconversion and/or significant rise (more than double) in the antibody titer; the borderline values for antibodies IgG phase 2 were $20-30 \mathrm{U} / \mathrm{ml}$.

Serum samples were taken from domestic sheep and goats by veterinary inspection and the serologic diagnosis was performed at the Croatian Veterinary Institute in Zagreb, by complement fixation test (CFT) at dilution of $1: 10$. Antibody titers of 10 to 40 suggest a latent Coxiella burnetii infection.

\section{RESULTS}

\section{Clinical Data}

The mean age of the hospitalized patients was 35.7 years, most of them, $91 \%$, were men. All patients had a pulmonary form of the disease and $85 \%$ had unilateral single opacities on chest $\mathrm{x}$-ray (Table $\mathbf{2}$ ). 
Table 2. Demographic and Clinical Baseline Characteristics of 197 Hospitalized Patients with Q Fever Pneumonia

\begin{tabular}{|c|c|}
\hline Characteristics & No (\%) of Patients \\
\hline \hline Mean age (yr/range) $35.7(16-72)$ & $180(91.4)$ \\
\hline Male & $17(8.6)$ \\
\hline Female & \\
\hline Risk factors & $165(83.7)$ \\
\hline Rural area & $32(16.3)$ \\
\hline Urban area & $167(85)$ \\
\hline Radiographic findings & $30(15)$ \\
\hline Single opacity & \\
\hline Multiple opacities & \\
\hline
\end{tabular}

Fig. (2). shows annual incidence of hospitalized patients with Q fever before, during and after the outbreaks. The persistance of higher incidence after the outbreaks in the main outbreak focus, the Zadar County, in comparison with the other two counties must be pointed out

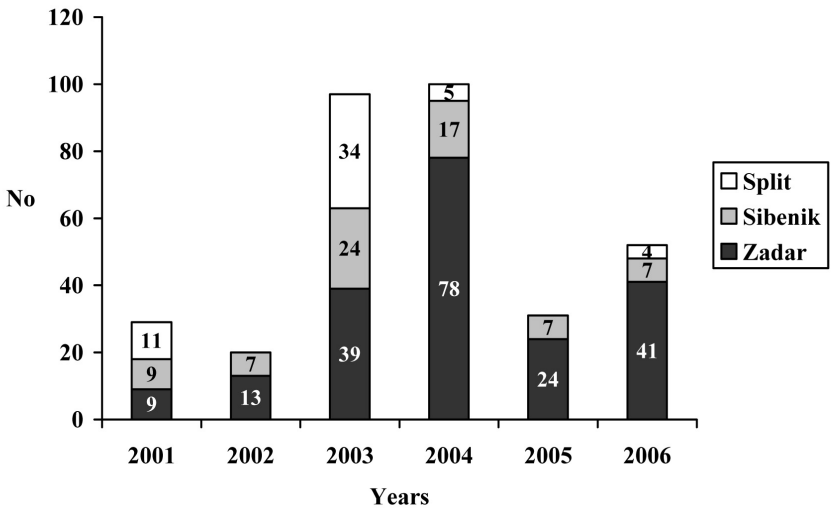

Fig. (2). Patients with $Q$ fever hospitalized in three Dalmatian hospitals from 2001 to 2006 .

\section{Meteorological Data}

Along the eastern coast of the Adriatic Scirocco (Jugo) and Bora are the prevailing winds; in North and Mid Dalmatia they represent $70-80 \%$ of all winds (Fig. 1). The strongest winds blow mainly in winter.The Bora is a relatively cold dry wind, blowing from ENE direction with a mean speed of $3-6 \mathrm{~m} / \mathrm{s}$ but reaching $15 \mathrm{~m} / \mathrm{s}$ at some locations; $5.5-10.7 \mathrm{~m} / \mathrm{s}$ speed is considered the moderate Bora. The Scirocco is a moderate, sometimes stormy wind, blowing from SE direction with a typical speed of $10 \mathrm{~m} / \mathrm{s}$, occuring most frequently in spring and autumn and usually accompanied by precipitation. Both winds are transient ocurrences with a typical duration of several days [5].

During the period from January 2002 to December 2004, there were no significant deviation in the usual climatologic characteristics either one month before (December) or during the Q fever outbreaks. In January 2003, winds with weak to moderate speed dominated, the maximum Bora speed of 6.7 $\mathrm{m} / \mathrm{s}$ was measured on January 24 and that of Scirocco of 12.3 $\mathrm{m} / \mathrm{s}$ on January 21. In February 2004 weak Bora dominated, with maximum speed of $9.3 \mathrm{~m} / \mathrm{s}$ on February 12; the maximum speed of Scirocco was $12.3 \mathrm{~m} / \mathrm{s}$ on January 22 .

Along the eastern coast of the Adriatic, the average anual rate of precipitation is $750-1000 \mathrm{~mm}$, mostly during autumn and winter months ( Dalmatia is an area with the lowest annual rate of rainy days in Croatia).

During the period of study, December 2002 and January 2003 were much more rainy than February, March and April 2003, with 237.1 and $36.6 \mathrm{~mm}$ of precipitation, respectively. In December 2003 and January 2004, the precipitation of only $136 \mathrm{~mm}$ was registered, while the period from February to April was much more rainy with $232.2 \mathrm{~mm}$ of rain. Precipitation rate in the years preceeding (2001-2002) and following (2005-2006) the outbreak years showed however, only small deviations with regard to the months of outbreaks. The average precipitation rate during these years was 62 $\mathrm{mm}$, while monthly precipitation rarely exceed $150 \mathrm{~mm}$, even during the autumn or winter.

\section{Serosurveillance in Sheep and Goats}

Until the outbreaks described there was no systematic serologic survey of coxiellosis in domestic animals in Croatia.

During the first outbreak in 2003, antibodies to Coxiella burnetii were detected in 129 (10.8\%) of 1196 animals analyzed (920 sheep and 276 goats) on 12 farms around the outbreak focus where road-workers were affected (Fig. 1: Obrovac area); goats were more often affected than sheep (31.2\% vs $4.7 \%$;). In 2004, only 182 sheep from pastures close to the mentioned aluminium factory in suburban Zadar area (Fig. 1: Murvica area) were analyzed and $11 \%$ of them were seropositive; 11 employees from this factory got Q fever pneumonia. Table $\mathbf{3}$ shows distribution of antibodies to Coxiella burnetii among domestic animals during the outbreaks.

Table 3. Prevalence of Antibodies to Coxiella burnetii in Sheep and Goats in 13 Farms in North Dalmatia, 2003/4

\begin{tabular}{|c|c|c|}
\hline \multirow{2}{*}{ Animal } & \multicolumn{2}{|c|}{ No of Samples / \% of Positive CFT* } \\
\cline { 2 - 3 } & $\mathbf{2 0 0 3}$ & $\mathbf{2 0 0 4}$ \\
\hline \hline Sheep & $920 / 4.7$ & $182 / 11.0$ \\
\hline Goat & $276 / 31.2$ & - \\
\hline
\end{tabular}

${ }^{*} \mathrm{CFT}=$ complement fixation test

It is of interest to note that the seroprevalence of the antibodies was highest $(>10 \%)$ in animals grazing on pastures almost $30 \mathrm{~km}$ east and west from the outbreak center (Fig. 1: Obrovac area, the outer circles) and very low, about $1 \%$ in the center (Fig. 1: Murvica area).

According to data from the Croatian Veterinary Institute for the period from 2001 to 2006, there were no greater 
variations in seroprevalence of Coxiella burnetii antibodies among domestic animals in Croatia, with the mean annual rate of $1.7 \%$. Also, there were no greater increase in animal illnesses or abortions and importation of new ruminants from neighboring countries (Annual reports, 2001-2006, Croatian Veterinary Institute, Zagreb). Here we must point out that sheep and goat farms are much more frequent in the southern part of Croatia than in the rest of the country which could explain the difference in seroprevalence of animal antibodies in Dalmatia.

\section{DISCUSSION}

During recent years several outbreaks of $Q$ fever have been described in Europe [6-11].

In Dalmatia and some other parts of Croatia Q fever has been recognized for a long time as an occupational disease occuring usually sporadically during the first few months of the year. Most of the cases came from the old and wellknown Q fever which is already mentioned: Gala in the Split County, Drnis-Siveric area in the Sibenik County and Obrovac-Murvica area in the Zadar County. The only relevant seroepidemiologic study was done in the Zadar county showing an anamnestic antibody titer to Coxiella burnetii (by IFA method) in as many as $37 \%$ of the residents [12]. However, no greater outbreaks had been registered in Dalmatia until recently.

The first Q fever outbreak occured in Dalmatia in 2003, the second in 2004. The first one consisted of three waves involving three different counties - Zadar, Sibenik and Split and lasting for the first few months of 2003 with focuses quite faraway from one another. Our epidemiological and veterinary data showed no connection between the three areas except for the 40 road-workers who worked during the outbreak in the same place at the same time; they were hospitalized in the three different county hospitals depending on their place of residents.

In 2004, a bigger outbreak occured only in the Zadar county; in the Sibenik county a minor outbreak originated from the same Siveric-Drnis focus as in 2003, while in the Split county only a few cases of $\mathrm{Q}$ fever pneumonia were registered.

Each area where outbreaks occured is known as an old Q fever focus (Fig. 1) characterized by traditional sheep and goat breeding, with sporadic cases of human $Q$ fever registered in the past, mostly among residents of rural areas in close contact with domestic animals or animal products.

In 2003 and 2004, before and throughout the outbreak periods, there were no unusual number of animal illnesses or abortions during the lambing and goating seasons. Also there were no data on importation of new domestic ruminants on the farms. The humans affected were generally middle aged, mostly male (the traditional way of life in Dalmatia shearing, slaughtering and skinning animals - puts them to a much greater exposure to Coxiella reservoirs) and residents of the epidemic focus area or its vicinity, except for the roadworkers mentioned above. Other affected people lived in various urban places, some of them visiting the foci for professional or other reasons, but some of them did not go outside the cities at all.
Radiographic findings of the most commonly recorded unilateral single area of opacity were described earlier and support the view that radiographic differentiation of $Q$ fever from the other community-acquired pneumonia is not possible [13].

It is our opinion that the outbreaks could be primarily related to lambing and goating period which in Dalmatia is mostly $(70-80 \%)$ from late December to May, and reaches its peak in February. This is supported by the facts that animals rarely develop illness after Coxiella infection, that infection of domestic ruminants is usually seasonal, and that temporal distribution of infection in humans is mainly related to lambing (goating, calving) season. It is also well known that Coxiella can survive in the environment for months and can be transmitted long distances by aerosols, particularly on windy days. Finally, our seroprevalence study showed that a much greater proportion of domestic ruminants is infected with Coxiella in Dalmatia than in other parts of the country.

Sporadic cases and rare small familial outbreaks could be related to slaughterhouses, which are very often in the open area and without veterinary control. Besides, it is important to mention that animals are killed throughout the year, but mostly from February to June, and that sometimes it happens daily, i.e. according to customers' need.

The occurence of outbreaks during the successive years at the same areas can be explained by the possibility of C.burnetii persistance in domestic ruminants $[14,15]$ and/or by the organism maintenance in nature in a circle between ticks, wild and domestic animals [16]. Alternatively, it is possible that there was a concurrent introduction of new strains of organism to several farms in one area ; to test this hypothesis the strains identification is necessary.

A number of the affected people, $11(10.7 \%)$ in 2003 and $21(21.0 \%)$ in 2004 , live, as mentioned above, strictly in the urban centers with no data of connection with any infection source. Some reports suggest the dominant epidemiologic role of wind in Q fever outbreaks, particularly in the case of urban infections [17-19], but our findings could not rule out other possibilities as well.

Firstly, in 2003 farms with the most infected sheep and goats were faraway, more than $20 \mathrm{~km}$ to the east and west from the outbreak center with affected roadworkers and also from the cities. This distance is more than the maximum one measured from the source of contamination in some earlier observations $[17,18]$. However, before and during these outbreaks a moderate bora dominated.

During the 2004 outbreak, the same climatologic picture with weak winds was present. In the case of the small outbreak with 11 affected factory workers there was no need for wind transmission because their factory was located on a farm with infected sheep ( $11 \%$ of them were seropositive).

In Dalmatia, the sheep and goat farms are often located in suburban areas which increases the possibility of even weak winds transmitting contaminated aerosols and contributing to urban infections. Other possibilities in this urban spread of $Q$ fever include: the vicinity of a number of slaughterhouses $[20,21]$, farm vehicles carrying contaminated straw and manure [22], reservoirs like dogs and cats (which in Dalmatian towns are traditionally numerous), the wild brown 
rat (which can transmit infection between farm animals and cats) [23] and even pigeons [24].

Apart from the wind influence, the analysis of the meteorologic data during 2003 and 2004 showed an association between rainfall and the incidence of $\mathrm{Q}$ fever, i.e. the disease predominantly occured during the rainy period in both years. Similar observations in French Guiana led to the speculation that a wild reservoir is responsible for transmission [25].

To summarize, the feature of $Q$ fever in Dalmatia confirms the known fact that the $\mathrm{Q}$ fever epidemiology is a result of multifactorial influences depending primarly on dynamic interrelationship between animal reservoirs (which are showing a rising spectrum), time when livestock give birth, atmospheric conditions and host immunity. The only predictable factors of $\mathrm{Q}$ fever in Dalmatia seem to be the season and the precipitation rate. The seropositivity rate in animals seems to be of variable quality and to help in the case of Q fever outbreak, as was shown earlier [26]; besides, seronegative animals can also shed bacteria [27]. Also, the wind showed to be of variable influence in the case of outbreak. The unpredictable rise of urban cases of $Q$ fever finalizes the number of questions for future research.

\section{REFERENCES}

[1] Borcic B, Galinovic-Weisglass M, Aleraj B, Soic-Kosic N, Delimar N. Epidemic of Q fever in North Croatia in 1983 (epidemiological and serologic characteristics). Lijec Vjesn 1984; 10: 353-7.

[2] Dzelalija B, Morovic M, Stankovic S, Vukic L, Dobec M, Dujella J. Clinical spectrum of rickettsioses in the Zadar area. Lijec Vjesn 1990; 112: 102-5.

[3] Milotic I, Miletic B, Morovic M. Clinical, epidemiological and epizootic features of Q fever in the Norther Coastal part of Croatia from 1989 to 1998. Acta Med Croat 2001; 55: 53-7.

[4] Morovic M, Dzelalija B, Novakovic S, Stankovic S, Dujella J. Clinical spectrum of rickettsioses in the North-Dalmatian subregion. In: Kazar J, Raoult D, Eds. Rickettiae and rickettsial diseases. Proceedings of the IVth International Symposium; 1990 Oct 1-6; Piestany Spa; Publishing House of the Slovak Academy of Sciences: Bratislava 1991; 540-5

[5] Pandzic K, Likso T. Eastern Adriatic typical wind fielr patterns and large-scale atmospheric conditions. Int J Climatol 2005; 25(1): 8198.

[6] Winner SJ, Eglin RP, Moore VIM, Mayon-White RT. An outbreak of Q fever affecting postal workers in Oxfordshire. J Infect 1997; 14: 255-61.

[7] Smith DL, Ayres JG, Blair I, et al. A large Q fever outbreak in the West Midlands: clinical aspects. Resp Med 1993; 87: 509-16.

[8] Selvaggi MT, Rezza G, Scagnelli M, et al. Investigation of a Q fever outbreak in norther Italy. Eur J Epidemiol 1996; 12: 403-8.
[9] Hellebrand W, Breuer T, Peterson L. Changing epidemiology of Q fever in Germany, 1947-1999. Emerg Infect Dis 2001; 7: 789-96.

[10] Raoult D, Mege JL, Marrie T. Q fever: Queries reamining after decades of research. In: Scheld WM, Craig WA, Hughes JM, Eds. Emerg infect Washington D.C., ASM Press 2001; 29-56.

[11] van Woerden HC, Mason BW, Nehaul LK, et al. Q fever outbreak in industrial setting. Emerg Infect Dis 2004; 10(7): 1282-9.

[12] Dzelalija B, Morovic M, Novakovic S. Rickettsial antibody in North Dalmatia: Antibodies to R. conorii, R. akari, R. slovaca and C. burnetii among urban and rural population. In: Kazar J, Raoult D, Eds. Rickettsiae and rickettsial diseases. Proceedings of the IVth International Symposium; 1990 Oct 1-6; Piestany Spa: Publishing House of the Slovak Academy of Sciences Bratislava 1991; 45764.

[13] Gikas A, Kofteridis D, Bouros D, Voloudaki A, Tselentis Y, Tsaparas N. Q fever pneumonia: Appearance on chest radiographs. Radiology 1999; 210: 339-43.

[14] Biberstein EL, Behymer DE, Bushnell R, Crenshaw GL, Reinmann HP, Franti CE. A survey of Q fever (Coxiella burnetii) in Californian dairy cows: results of field trials. Am J Vet Res 1974; 35: 1577-82.

[15] Berri M, Souriau A, Crosby M, Rodolakis A. Shedding of Coxiella burnetii in ewes in two pregnancies following an episode of Coxiella abortion in a sheep flock. Vet Microbiol 2002; 85: 55-60.

[16] Woldehiwet Z. Q fever (coxiellosis): epidemiology and pathogenesis. Res Vet Sci 2004; 77: 93-100.

[17] Hawker JI, Ayres JG, Blair I, et al. A large outbreak in the West Midlands: winborn spread into a metropolitan area? Commun Dis Public Health 1998; 1(3): 180-7.

[18] Tissot-Dupont H, Torres M, Nezri M, Raoult D. Hyperendemic focus of Q fever related to sheep and wind. Am J Epidemiol 1999; 150: 67-74.

[19] Tissot-Dupont H, Amadei M-A, Nezri M, Raoult D. Wind in November, Q fever in December. Emerg Infect Dis 2004; 10(7): 1264-9.

[20] Gilroy N, Formica N, Beers M, Egan A, Conaty S, Marmion B. Abattoir-associated Q fever: a Q fever outbreak during a Q fever vaccination program. Aust N Z J Public Health 2001; 25: 362-7.

[21] Brouqui P, Badiaga S, Raoult D. Q fever outbreak in homeless shelter. Emerg Infect Dis 2004; 10(7): 12979.

[22] Salmon MM, Howels B, Glencross EJG, Evans AD, Palmer SR. Q fever in an urban area. Lancet 1982; 1(8279): 1002-4.

[23] Webster JP, Lloyd G, Macdonald DW. Q fever (Coxiella burnetii) reservoir in wold brown rat /Rattus norvegicus) populations in the UK. Parasitol 1995; 110: 31-5.

[24] Stein A, Raoult D. Pigeon pneumonia in Provence. A bird borne Q fever outbreak. Clin Infect Dis 1999; 29: 617-20.

[25] Gardon J, Heraud J-M, Laventure S, et al. Suburban transmission of Q fever in French Guiana: Evidence of a wild reserovoir. J Infect Dis 2001; 184: 178-84

[26] Parker NR, Barralet JH, Bell AM. Q fever. Lancet 2006; 367: 67988.

[27] Berri M, Souriau A, Crosby M, Rodolakis A. Shedding of Coxiella burnetii in ewes in two pregnancies following an episode of Coxiella abortion in a sheep flock. Vet Microbiol 2002; 85: 55-60. 